\title{
$X V$. On the significance of Einstein's gravitational equations in terms of the curvature of the world
}

\section{A.S. Eddington M.A. F.R.S.}

To cite this article: A.S. Eddington M.A. F.R.S. (1922) XV. On the significance of Einstein's gravitational equations in terms of the curvature of the world, Philosophical Magazine Series 6, 43:253, 174-177, DOI: $10.1080 / 14786442208633860$

To link to this article: http://dx.doi.org/10.1080/14786442208633860

曲 Published online: 08 Apr 2009.

Submit your article to this journal $[\pi$

Цll Article views: 6

Q View related articles $\square$

Citing articles: 1 View citing articles 5 


\section{Prof. Eddingten: Significance of Einstein's Gravitational}

effects are due to contaminations, chiefly by oxidation, of the surfaces of the tungsten filament and the copper anode. The extreme variations which have been observed for the position of the voltage at which the current attained half the saturation value are -2.11 and +0.17 ; the corresponding curves are shown in fig. 7. This corresponds to a total change in the contact potential difference of 2.28 volts. The extreme displacements in the positive direction along the voltage axis, such as that with the half saturation value at +.17 in fig. 7 , were obtained when the tube had been well baked out and the tungsten filament had been intentionally oxidized by glowing in air at a low pressure with the tube cold before testing. The evidence seems clear that an oxilized filament is electropositive by about $0 \cdot 6$ volt as compared with a clean filament at these temperatures.

When the filaments were oxidized the currents were found to be difficult to saturate. This is shown by the upward slope of the relatively flat part of the curve on the right in tig. 7. There seems to be no doubt that this upward slope is due to the oxidized conditions of the tungsten surface. On glowing out the tungsten at a high temperature so as to cause the layer of oxide to evaporate, curves such as B fig. 7 were obtained. These are displaced about 0.6 volt to the left, corresponding to the more electronegative character of the clean tungsten, and at the same time the upward slope of the saturation part is found gradually to disappear.

XV. On the significance of Einstein's Gravitational Equations in terms of the Curvature of the World. By A. S. Eddingros, M.A., F.R.S., Plumian Professor of Astronomy in the Unicersity of Cambridge *

$\mathrm{T}$ rhe Appendix to the paper on "The Relativity of Field and Matter" $\dagger, I$ gave a proof of the theorem that Einstein's equations $G_{\mu \nu}=\lambda g_{\mu \nu}$ express the condition that the radius of curvature of sections of the world is the same at all points and for all directions. I have since found that this proof is not sufficiently general. I took too limited a view of the vagaries of which a four-dimensional surface is capable when it has six extra dimensions to twist about in. I assumed that, although ten dimensions of Euclidean space are necessary for the representation of a four-dimensional Riemannian space, a small portion of the latter near the

* Coumunicated by the Author.

† Phil. Mag. November 1921, p. 800. 
origin, for which cubes of the coordinates are negligible, might be represented in five dimensions; but this is not generally true, and unfortunately the simplification is not admissible in the cases of greatest practical importance, $e . g$. the gravitational field of the sun. The theorem itself is correct; but it requires a more general proof.

Take a point on the four-dimensional surface as origin. Let $\left(x_{1}, x_{2}, x_{3}, x_{4}\right)$ be rectangular coordinates in the tangent plane at the origin. The normal is a six-dimensional continuum in which we take rectangular axes $z_{1}, z_{2} \ldots z_{6}$. Six equations will be necessary to define the fonr-dimensional surface, and at a regular point these may be taken to be

$$
2 z_{r}=a_{r \mu \nu} x_{\mu} x_{\nu}+\text { higher powers } \quad(r=1,2 \ldots 6) .
$$

There are no linear terms in $x_{\mu}$ since deviations perpendicular to the tangent plane are of the second order compared with displacements parallel to the tangent plane. According to the usual summation convention (applying only to Greek suffixes) $a_{r_{\nu}} x_{\mu} x_{\nu}$ is summed for values of $\mu$ and $\nu$ from 1 to 4 , and represents a general quadratic function of the $x$ 's. By differentiation

$$
d z_{r}=a_{\mu_{\mu} \nu} x_{\mu} d x_{\nu} ; d z_{r}^{2}=a_{\mu \mu \nu} x_{\mu} d x_{\nu} \cdot a_{r_{\sigma \tau} x_{\sigma}} d x_{\tau} .
$$

Since the geometry of the ten-dimensional space is Euclidean,

$$
\begin{aligned}
-d s^{2} & =d x_{1}^{2}+d x_{2}^{2}+d x_{3}^{2}+d x_{4}^{2}+d \varepsilon_{1}^{2}+\ldots+d{\tilde{\sigma_{6}}}^{2} \\
& =d u_{1}^{2}+d x_{2}^{2}+d x_{3}^{2}+d x_{4}^{2}+\underset{r}{2}\left(a_{r_{\nu} \nu} a_{r \sigma \tau} x_{\mu} x_{\sigma}\right) d x_{\nu} d x_{\tau}+\text { terms }
\end{aligned}
$$

containing cubes of the coordinates.

Equating this to $-g_{\mu \nu} d x_{\mu} d x_{\nu}$, we have at the origin

$$
g_{11}=y_{22}=g_{33}=g_{44}=-1, \quad g_{12}=0 \text {, etc. }
$$

All first derivatives of the $g_{\mu \nu}$ vanish.

The second derivatives are given by

$$
\frac{\partial^{2} g_{\nu \tau}}{\partial x_{\mu} \partial x_{\sigma}}=-\sum_{r}\left(a_{r_{\mu \nu}} a_{r \sigma \tau}+a_{r \mu \tau} a_{r \sigma \nu}\right)
$$

When the first derivatives vanish, the Riemann Chrietofiel tensor simplifies to

$$
\begin{aligned}
\mathrm{B}_{\mu \nu \sigma \rho} & =\frac{1}{2}\left(\frac{\partial^{2} g_{\sigma \rho}}{\partial x_{\mu} \partial x_{\nu}}+\frac{\partial^{2} g_{\mu \nu}}{\partial x_{\sigma} \partial x_{\rho}}-\frac{\partial^{2} g_{\mu \sigma}}{\partial x_{\nu} \partial x_{\rho}}-\frac{\partial^{2} g_{\nu \rho}}{\partial x_{\mu} \partial x_{\sigma}}\right) \\
& =\sum_{r}\left(a_{r_{\mu \nu}} a_{r_{\sigma \rho}}-a_{r_{\mu \sigma}} a_{\nu \rho}\right) .
\end{aligned}
$$

It will be seen that the six $\tilde{z}_{r}$ 's contribute terms to $\mathrm{B}_{\mu \nu \sigma \rho}$ which are simply additive. Our subsequent formulie will 


\section{Significance of Einstein's Gratitational Equations.}

involve $B_{\mu \nu \sigma \rho}$ linearly; so we need consider only one $z$ at a time, and shall accordingly shorten the notation by dropping the $r$. Thus

Hence

$$
B_{\mu \nu \sigma \rho}=a_{\mu \nu} a_{\sigma \rho}-a_{\mu \sigma} a_{\nu \rho} .
$$

$$
\mathrm{G}_{\mu \nu}=g^{\sigma \rho} \mathrm{B}_{\mu \nu \sigma \rho}=-a_{\mu \nu}\left(a_{11}+a_{22}+a_{33}+a_{44}\right)+a_{\mu \sigma} a_{\nu \sigma}
$$

by substituting the Euclidean values of the $g$ 's at the origin given above.

In particular

$$
\begin{aligned}
\mathrm{G}_{11} & =-a_{11}\left(a_{11}+a_{22}+a_{33}+a_{44}\right)+a_{11}{ }^{2}+a_{12}{ }^{2}+a_{13}{ }^{2}+a_{14}{ }^{2} \\
& =\left(a_{12}{ }^{2}-a_{11} a_{22}\right)+\left(a_{13}{ }^{2}-a_{11} a_{33}\right)+\left(a_{14}{ }^{2}-a_{11} a_{44}\right) . .
\end{aligned}
$$

Also

$$
\begin{aligned}
\mathrm{G}=g^{\mu \nu} \mathrm{G}_{\mu \nu} & =\left(a_{11}+a_{22}+a_{33}+a_{44}\right)^{2}-\left(a_{11}{ }^{2}+a_{22}{ }^{2}+\ldots+2 a_{12}{ }^{2}+\ldots\right. \\
& =-2\left\{\left(a_{12}{ }^{2}-a_{11} a_{22}\right)+\ldots(\text { six terms })\right\} . \quad \text {. . }
\end{aligned}
$$

Hence, since $g_{11}=-1$,

$$
\begin{array}{r}
\mathrm{G}_{11}-\frac{1}{2} g_{11} \mathrm{G}=-\left\{\left(a_{23}{ }^{2}-a_{22} a_{33}\right)+\left(a_{24}{ }^{2}-a_{22} a_{44}\right)\right. \\
\left.+\left(a_{34}{ }^{2}-a_{33}{ }^{a_{44}}\right)\right\} . \quad .
\end{array}
$$

Consider the three-dimensional continuum, which is the section of the world by the plane $x_{1}=0$. This is described by three coordinates $x_{2}, x_{3}, x_{4}$ and its Gaussian curvature, which we shall denote by $G_{(1)}$, is formed by dropping the terms in $\mathrm{G}$ which contain the suffix 1 . We see by (2) and (3) that

$$
\frac{1}{2} \mathrm{G}_{(1)}=\mathrm{G}_{11}-\frac{1}{2} g_{11} \mathrm{G} \text {. . . . . }
$$

Since the $\approx_{r}$ 's contribute linearly to each term in this equation, it holds for six $z$ 's as well as for one $z$.

The radius of (spherical) curvature of a manifold is defined as the radius of a sphere which has the same Gaussian curvature as the manifold. It is easily shown that for the Gaussian curvature $G_{(1)}$ of a three-dimensional manifold, the corresponding radius $\rho_{1}$ is given by

$$
G_{(1)}=\frac{6}{\rho_{1}^{2}} \text {. }
$$

Thus the result (4) may be written

$$
\mathrm{G}_{11}-\frac{1}{2} g_{11} \mathrm{G}=\frac{3}{\rho_{1}^{2}} . \quad \text {. . . . }
$$

Consider the quadric

$$
\left(G_{\mu \nu}-\frac{1}{2} g_{\mu \nu} G\right) d x_{\mu} d x_{\nu}=3 . \quad \text {. . . . }
$$


Setting $d x_{\mu}=\left(\rho_{1}, 0,0,0\right)$ we see by (5) that the equation is satisfied; that is to say, $p_{1}$ is the radius of the quadric in the $x_{1}$ direction. The quadric is seen by inspection of its equation to be invariant; consequently we may take $x_{1}$ in any direction we please. Hence the radius of the quadric (6) in any direction is equal to the radius of (spherical) curvature of the corresponding section of the world.

If $\mathrm{G}_{\mu \nu}=\lambda g_{\mu \nu}$, then $\mathrm{G}=4 \lambda$, and the quadric reduces to

$$
\begin{array}{ll} 
& -\lambda g_{\mu \nu} d x_{\mu} d x_{\nu}=3 \\
\text { or } \quad & -d s^{2}=3 / \lambda,
\end{array}
$$

showing that the quadric is a sphere of radius $\sqrt{ }(3 / \lambda)$. Conversely, if the radii of spherical curvature of sections of the world at all points and in all directions are equal to $\sqrt{ }(3 / \lambda)$, Einstein's equations $G_{\mu \nu}=\lambda g_{\mu \nu}$ will be satisfied. This demonstrates the theorem.

It may be noticed that in this general proof we have substituted spherical curvature for the normal curvature considered in the Appendix to the previous paper. This is necessary because in the general case normal curvature becomes meaningless.

XVI. On a Type of Oscillation-Hysteresis in a Simple Triode Generator. By E. V. Appleton, M.A., M.Se., Fellow of St. John's College, Cambridge, and BALTH. VAN DER POL junr., D.Sc., Conservator Physical Laboratory of 'Teyler's Institute, Haarlem (Holländ)*

7 HE conditions for the production of iree infinitesimal oscillations in various triode circuits have been worked out in great detail during the last few years, but the question of the stability and maintenance of oscillations of finite amplitude does not appear to have received equal attention. In a recent paper $\dagger$ we have dealt with the calculation of the amplitude finally attained in a simple case of free triode vibrations in which use was made of a non-linear "oscillation characteristic" easily determined by experiment for any particular tube and circuit. This oscillation characteristic, which represents the relation between the variations of anode potential and of anode current, may be regarded as expressing the electrical properties of an imaginary non-reactive resistance connected in parallel with the inductance of the

- Communicated by Professor Dr. H. A. Lorentz, For.Mem.R.S.

$\dagger$ Phil. Mag, vol. xlii. p. 201, August 1921.

Phil. Mag. S. 6. Vol. 43. No, 253. Jan. 1922. 